\title{
EDITORIAL
}

\section{Interventions for smoking cessation: the outcomes are determined by the questions asked}

\author{
R.P. Murray*, N.R. Anthonisen ${ }^{+}$
}

There is an increasing awareness of the health risks associated with smoking among the general public. However, the rates of smoking in Western countries have been remarkably resistant to change, and in developing countries the rates are increasing. Many interventions of the health promotion variety are being explored, while the tobacco industry is just as interested in developing more effective techniques to promote smoking.

There have been vigorous attempts to find the optimum ways to help people quit. There is a broad awareness among professionals that interventions to help people who want to stop smoking benefit from both behavioural and pharmacological components. In the current decade, most of the advances have been pharmacological.

A decade ago, interventions that were primarily behavioural were predominant. For example, a meta-analysis by VISWESVARAN and SCHMIDT [1], included the results of 633 studies conducted before nicotine replacement was commonly available. Nicotine replacement (nicotine gum) was used in $6 \%$ of these studies. The most effective interventions were multi-component programmes $(35 \%$ success rate), hypnosis (36\%), smoke aversion techniques (31\%), group withdrawal clinics (30\%), and acupuncture (30\%). The least effective were self-care $(15 \%)$, nicotine gum $(16 \%)$, and physician advice $(7 \%)$. Cessation results for controls averaged $6 \%$. Subsequent meta-analyses have more often focused on the use of nicotine replacement therapies. CEPEDA-Benito [2], in a meta-analysis of 33 studies of the efficacy of nicotine gum, found that the only studies where the gum affected success in the long-term were those where it had been combined with intensive behavioural treatments. SILAGy et al. [3] considered the results of 53 trials involving nicotine replacement therapy and concluded that it was effective in different forms, but largely independent of the intensity of additional support provided. FIORE et al. [4] included 17 nicotine patch efficacy studies that were double-blind, placebo-controlled with random assignment of subjects. They found that active patch subjects were more than twice as likely to quit smoking as were participants using a placebo patch. This effect was present at both high and low intensities of counselling. Intensive behavioural counselling had a modest positive impact on quit rates, which was stronger

\footnotetext{
*Dept of Community Health Sciences and ${ }^{+}$Faculty of Medicine, University of Manitoba, Winnipeg, Manitoba, Canada

Correspondence: R.P. Murray, Dept of Community Health Sciences, Room MS-754, 820 Sherbrook St., Winnipeg, Manitoba, R3A 1R9,
} Canada. Fax: 12047874547. at the end of treatment than after six months. In the metaanalysis conducted by LI WAN Po [5], the extent of behavioural support in each study is described, but it is not included as a variable in the meta-analysis.

It is easy to conclude from the more recent of these reports that the behavioural counselling component of these programmes is largely ineffectual. However, nicotine patch studies are often funded by the industry that manufactures the patch, which has a relatively small interest in demonstrating the contribution of behavioural interventions. Furthermore, investigators often appear to emulate the level of counselling that would be delivered in a physician's office, and it is unlikely that intensive counselling would be offered in such a setting.

Studies have compared various nicotine delivery systems, various concentrations of nicotine, and the combination of nicotine with several other agents. Such studies are both time consuming and expensive to conduct, but are necessary in order to gauge the progress made by current revisions to "best practice" in the area of interventions for smoking. The results reported by TøNNESEN et al. [6] in this issue of the European Respiratory Journal add to this literature.

In the TøNNESEN et al. [6] Collaborative European AntiSmoking Evaluation (CEASE) trial, the investigators at 36 clinical centres collaborated to provide results from 3,575 adults who were attempting to stop smoking. Participants were given 25 or $15 \mathrm{mg}$ or placebo patches, for 8 or 22 weeks in a randomized double-blind design. The rate of sustained smoking cessation was followed for $1 \mathrm{yr}$. The investigators found that dose was related to cessation success, but duration of treatment was not.

In the CEASE trial, there was a 10-fold difference between cessation rates in the active and placebo groups in the best of their clinics as compared to their least successful clinic. This may either be due to clinic differences at baseline or to different effects of the intervention in the clinics. It would be very interesting to rank the intensity of behavioural intervention in these clinics, and then assess whether clinics divided into quintiles of intensity of the behavioural programmes would find that intensity was reflected in their mean success rates.

TøNNESEN et al. [6] report 12-month sustained cessation rates of around $16 \%$ with the $25 \mathrm{mg}$ patch, $13 \%$ with the 15 mg patch, and $10 \%$ with placebo. The Lung Health Study, for example, reported 12-month sustained cessation rates, biochemically verified, of approximately $35 \%$ with ad libitum use of $2 \mathrm{mg}$ nicotine gum and intensive behavioural intervention, and $10 \%$ for usual care (controls) [7]. It is not possible to attribute the differences in cessation rates in the 
Lung Health Study to either the behavioural intervention or nicotine replacement. Nicotine replacement and the behavioural intervention are not independent of each other, since monitoring and instruction is required for the selfadministration of the nicotine replacement therapy. A comparison of these studies does suggest that an intervention which is designed to demonstrate the efficacy of nicotine replacement will likely understate the potential importance of other intervention components.

A pair of studies by FIORE et al. [8] addressed this issue. They tested nicotine patch with two counselling treatments, one intended to emulate what might be conducted in a clinician's office practice, and one an intensive treatment group approach. The intensive group approach had 6-month abstinence rates 2 to 3-times higher than the individual counselling approach. The difference was greatest among participants in each study receiving the placebo patch, suggesting that it was the counselling treatment which had the most influence on abstinence rates, rather that the patch. The authors concluded that there is no reason to limit the adjuvant treatment which accompanies the patch to brief individual therapy when group intervention is often less expensive and is apparently more effective

This evidence illustrates one of the subtle and probably unintentional ways that research sponsorship influences research results. In the CEASE trial, the concern of the sponsor (the manufacturer) about the effectiveness of the technology under study (the nicotine patch) results in a design that is optimized to demonstrate patch effectiveness. It accomplishes this in an apparently precise way. However, if readers wish to consider the importance of behavioural counselling as well (and this was also an element of the CEASE trial), then they would not find a comparable amount of attention given to it. This is a common void in studies and also in meta-analyses.

To return to the issue of why so many people still smoke, there are a number of factors at play. At present, general population rates of smoking are not clearly decreasing. What else can be done to enhance the spread of smoking cessation? Programmes to increase public aware- ness of the health risks of smoking and the corresponding benefits of quitting are now widespread. Nicotine dependence is clearly a key element in the maintenance of smoking, and nicotine replacement systems are widely available. The fact that this climate for smoking cessation has not produced greater success indicates that many people prefer to ignore the messages given them. There is a need to develop smoking cessation campaigns that depend less on the recipient's empathy with what we are trying to accomplish, and more on the aims that smokers may have for themselves.

\section{References}

1. Viswesvaran C, Schmidt FL. A meta-analytic comparison of the effectiveness of smoking cessation methods. J Appl Psychol 1992; 77: 554-561.

2. Cepeda-Benito A. Meta-analytical review of the efficacy of nicotine chewing gum in smoking treatment programs. $J$ Consult Clin Psychol 1993; 61: 822-830.

3. Silagy C, Mant D, Fowler G, Lodge M. Meta-analysis on efficacy of nicotine replacement therapies in smoking cessation. Lancet 1994; 343: 139-142.

4. Fiore MC, Smith SS, Jorenby DE, Baker TB. The effectiveness of the nicotine patch for smoking cessation: a meta-analysis. JAMA 1994; 271: 1940-1947.

5. Li Wan Po A. Transdermal nicotine in smoking cessation: a meta-analysis. Eur J Pharmacol 1993; 45: 519-528.

6. Tønnesen P, Paoletti P, Gustavsson G, et al. Higher dose nicotine patches increase one-year smoking cessation rates: results from the European CEASE trial. Eur Respir $J$ 1999; 13: 238-246.

7. Anthonisen NR, Connett JE, Kiley JP, et al. Effects of smoking intervention and the use of an inhaled anticholinergic bronchodilator on the rate of decline of FEV1: the Lung Health Study. JAMA 1994; 272: 1497-1505.

8. Fiore MC, Kenford SL, Jorenby DE, et al. Two studies of the clinical effectiveness of the nicotine patch with different counseling treatments. Chest 1994; 105: 524 533 . 